\title{
Especies vegetales que habitan en los derechos de vía de las carreteras principales de Nicaragua
}

\author{
J. Jiménez ${ }^{*}$ \\ Programa de Estudios Ambientales Urbanos Territoriales (PEAUT), \\ Universidad Nacional de Ingeniería (UNI) \\ PO Box 5595, Managua, Nicaragua \\ e-mail: julianaj3103@yahoo.com \\ (recibido/received: 03-Agosto-2009; aceptado/accepted: 21-Agosto-2009 \\ RESUMEN
}

\begin{abstract}
En este artículo se presenta información sobre las especies de árboles que se encuentran en los derechos de vía de las carreteras de Nicaragua, para lo cual se utilizó el inventario de árboles y arbustos en dos tramos de carreteras representativos del sistema ambiental dominante que se relacionan con vegetación en las carreteras y caminos que constituyen la red vial nacional. Con la información válida y un nivel de precisión satisfactorio, se establecieron los criterios de selección para elegir las carreteras en donde se estudiaron a las especies vegetales. Los aspectos analizados fueron abundancia, riqueza de las especies, grado de cubierta, dominancia, composición florística y vitalidad. Se concluye que las especies de árboles que habitan en las áreas verdes de los derechos de vía de las carreteras analizadas son especies ruderales, o remanentes de bosques riparios siendo frecuentes de encontrar: Tabebuia rosea (Bignoniaceae), Gliricidia sepium (Fabaceae), Azadirachta indica (Meliaceae), Guazuma ulmifolia (Sterculiaceae), Cordia alliodora (Boraginaceae), Senna siamea (Caesalpiniaceae), y Enterolobium cyclocarpum (Mimosaceae).
\end{abstract}

Palabras claves: abundancia; ambiente; riqueza de las especies; grado de cubierta; dominancia; composición florística; vegetación.

\begin{abstract}
The current article presents information about species of trees growing on the road easements on the highways of Nicaragua. Inventories of trees and bushes were carried out on two representative highways within the national roadway system to assess the most prevalent species of plants. The criteria for the selection of the highways under analysis were established by taking into account the relevant information on a precise and satisfactory level. The parameters analyzed were: proliferation, value of the species, degree of coverage, density, plant species composition and vitality. In conclusion, the tree species identified on the road easement areas were typically those of indigenous plants or the remains of riparian forests. The species most frequently found were Tabebuia rosea (Bignoniaceae), Gliricidia sepium (Fabaceae), Azadirachta indica (Meliaceae), Guazuma ulmifolia (Sterculiaceae), Cordia alliodora (Boraginaceae), Senna siamea (Caesalpiniaceae), y Enterolobium cyclocarpum (Mimosaceae).
\end{abstract}

Keywords: abundance; environment; richness of species; cover degree; dominance; plant species composition; flora.

\footnotetext{
*Autor para la correspondencia
} 


\section{INTRODUCCIÓN}

En Nicaragua existe escasa información oficial sobre las especies vegetales que pueblan el área del derecho de vía de las carreteras (MTI, 2000).

Sin embargo, la vegetación es uno de los principales indicadores de estado del ambiente, no sólo por el papel que desempeña este elemento como el productor primario de casi todos los ecosistemas, sino también por sus importantes relaciones con el resto de los componentes bióticos, abióticos, socioeconómicos y culturales del medio. La vegetación es estabilizadora de pendientes, retarda la erosión, influye en la cantidad y calidad del agua, mantiene microclimas locales, filtra la atmósfera, atenúa el ruido y es hábitat de especies de animales (Aguilo et. al, 2004).

En vista de la importancia del estudio de la vegetación el objetivo de este trabajo fue presentar información de las especies de árboles que habitan en dos tramos de carreteras ambientalmente representativos del 30\% de la red vial de Nicaragua.

\section{METODOLOGÍA}

\section{Selección de casos de estudios}

El estudio realizado fue de naturaleza descriptiva, en un universo de carreteras de 19,036 kilómetros de longitud que conforman la red vial de Nicaragua, tomando una muestra denominada red vial básica, la cual representa el $43 \%$ de las carreteras del país (MTI, 2005).

Se utilizaron cuatro momentos metodológicos para la selección de tramos representativos de la red vial de Nicaragua, que se designan de la siguiente manera: recopilación de información relativa a carreteras, análisis de los elementos ambientales de la red vial básica que influyen en la vegetación, definición de criterios de selección e identificación de tramos que cumplen con las características de selección.

Para esta tarea se emplearon las siguientes técnicas: recopilación y selección de documentos, análisis y procesamientos de datos, confección de matrices, establecimientos de criterios para seleccionar los dos tramos de carreteras que se usaron como casos de estudios, utilización de técnicas computarizadas y un conjunto de mapas en plataforma de sistema de información geográfica (programa Arc GIS, versión 8.2). a) Recopilación de información relativa a carreteras

Se determinó el área verde en los diferentes tipos carreteras, de conformidad a su clasificación funcional (MTI, 2000); lo que se ilustra en la tabla 1.

Tabla 1 Rango de ancho del área verde

\begin{tabular}{|l|l|}
\hline Derecho de vía $(\mathrm{m})$ & $\begin{array}{l}\text { Rango de ancho del } \\
\text { área verde }(\mathrm{m})\end{array}$ \\
\hline $\begin{array}{l}\text { Caminos vecinales con } \\
\text { ancho menor de 20 }\end{array}$ & $1-9$ \\
\hline $\begin{array}{l}\text { Carreteras colectoras y } \\
\text { troncales con ancho entre } \\
20-39\end{array}$ & $14-10$ \\
\hline $\begin{array}{l}\text { Carreteras troncales o } \\
\text { colectoras con ancho de } 40\end{array}$ & $34-30$ \\
\hline
\end{tabular}

b) Análisis de los elementos ambientales de la red vial básica que influyen en la vegetación

Se utilizaron de base los componentes y factores del ambiente establecidos en los manuales para realizar estudios ambientales en Latinoamérica conformando un modelo teórico de relaciones (Jiménez, 2008).

Se extrajeron las variables que tienen fuentes bibliográficas con mayor información histórica. Se identificaron las variables que cuentan con información válida.

Para la evaluación de los datos, se consideraron los siguientes elementos:

- Escala Geográfica: Si es la apropiada y que sea coherente con la información para el desarrollo del estudio

- Escala Temporal: Se refiere a la validez de la información demandada para efecto del estudio.

- Nivel de Agregación: la cantidad de información compilada.

Una vez identificados los elementos usados para la evaluación se discriminaron en: validez y precisión

c) Definición de criterios de selección

Se obtuvieron los componentes y factores que cuentan con información válida y con un nivel de precisión satisfactorio, en su mayoría con información histórica entre el período de 1971 al 2000. 
Se prestó relevancia a los ecosistemas y las formaciones vegetales utilizando el mapa elaborado por MARENA (2004), en la que se aplicó el sistema de clasificación fisionómica-ecológica de la vegetación de la tierra de Mueller-Dombois \& Ellemberg (1967), el cual es el sistema aceptado a nivel mundial por la UNESCO (1974). En la tabla 2, se reúnen los componentes y factores utilizados en la determinación de criterios de selección.

Tabla 2 Factores ambientales claves usados en la definición de criterios de selección

\begin{tabular}{|l|l|}
\hline Componentes & Factores \\
\hline $\begin{array}{l}\text { Medio } \\
\text { Biótico }\end{array}$ & $\begin{array}{l}\text { Ecosistemas y formaciones vegetales } \\
\text { (Unidad funcional básica) }\end{array}$ \\
\hline $\begin{array}{l}\text { Medio } \\
\text { Abiótico }\end{array}$ & $\begin{array}{l}\text { Topografía ( pendiente) } \\
\text { Clima (Temperatura y precipitación) } \\
\text { Suelo (Tipo de suelo) } \\
\text { Agua (Cuenca hidrográfica) }\end{array}$ \\
\hline $\begin{array}{l}\text { Medio socio- } \\
\text { económico y } \\
\text { cultural }\end{array}$ & $\begin{array}{l}\text { Carretera (área verde) } \\
\text { Pobreza (nivel de pobreza } \\
\text { municipal) } \\
\text { Poblados aledaños a las carreteras }\end{array}$ \\
\hline
\end{tabular}

En la tabla 3 se muestran los análisis resumidos en justificaciones y los criterios de selección para determinar la representatividad ambiental, relacionada con la vegetación en la red vial básica de Nicaragua.

Tabla 3 Síntesis de criterios de selección y su justificación

\begin{tabular}{|c|c|}
\hline Criterios de selección & $\begin{array}{l}\text { Justificación de la } \\
\text { representatividad }\end{array}$ \\
\hline $\begin{array}{l}\text { Localización dentro de } \\
\text { las precipitaciones } \\
\text { históricas de promedio } \\
\text { anual entre los } 1000 \text { - } \\
1800 \mathrm{~mm} \text {. }\end{array}$ & $\begin{array}{l}\text { El } 68 \% \text { de la red vial se } \\
\text { ubica en ese rango de } \\
\text { precipitaciones. }\end{array}$ \\
\hline $\begin{array}{l}\text { Localización en } \\
\text { temperaturas promedio } \\
\text { anuales que oscilan entre } \\
24-28^{\circ} \mathrm{C} \text {. }\end{array}$ & $\begin{array}{l}\text { El } 75 \% \text { de la red vial se } \\
\text { localiza en temperaturas } \\
\text { promedio entre } 24-28{ }^{\circ} \mathrm{C} \text {. } \\
\text { El } 20 \% \text { entre temperaturas } \\
\text { de } 22-24{ }^{\circ} \mathrm{C} \text {. }\end{array}$ \\
\hline $\begin{array}{l}\text { Ubicación al menos } \\
\text { sobre uno de los suelos } \\
\text { Inceptisol, Molisol ó } \\
\text { Ultisol. }\end{array}$ & $\begin{array}{l}67 \% \text { de la red vial se } \\
\text { encuentra en suelos: } \\
\text { Molisol (31\%), Ultisol } \\
(19 \%) \text { e Inceptisol (17\%). }\end{array}$ \\
\hline $\begin{array}{l}\text { Que las carreteras } \\
\text { oscilen en un rango de } \\
\text { pendientes entre } 0 \text { al } 5 \% \text {. }\end{array}$ & $\begin{array}{l}\text { El } 86 \% \text { de carreteras de } \\
\text { Nicaragua se ubican en } \\
\text { pendientes de } 0 \text { a } 5 \% .\end{array}$ \\
\hline
\end{tabular}

\begin{tabular}{|c|c|}
\hline Criterios de selección & $\begin{array}{l}\text { Justificación de la } \\
\text { representatividad }\end{array}$ \\
\hline $\begin{array}{l}\text { Que el derecho de vía } \\
\text { del tramo de carretera se } \\
\text { ubique aledaño a las } \\
\text { formaciones vegetales: } \\
\text { Sistemas agropecuarios } \\
\text { con } 10-50 \% \text { de } \\
\text { vegetación natural. }\end{array}$ & $\begin{array}{l}\text { El } 70 \% \text { de carreteras se } \\
\text { encuentran en sistemas } \\
\text { agropecuarios con } 10-50 \% \\
\text { de vegetación natural } \\
\text { (SPA1, } 56 \% \text { ), y sistemas } \\
\text { agropecuarios intensivos } \\
\text { (SPB, } 14 \% \text { ). }\end{array}$ \\
\hline $\begin{array}{l}\text { Localización en una de } \\
\text { las cuencas } \\
\text { hidrográficas: Río Coco } \\
\text { (No.45); río Grande de } \\
\text { Matagalpa (No. 55) y río } \\
\text { San Juan de Nicaragua } \\
\text { (No.69) }\end{array}$ & $\begin{array}{l}\text { El 64\% de las carreteras se } \\
\text { ubican en cuencas } \\
\text { hidrográficas: Río San } \\
\text { Juan (31\%), Río Coco } \\
\text { (18\%), Río Grande de } \\
\text { Matagalpa (15\%) }\end{array}$ \\
\hline $\begin{array}{l}\text { Que al menos el camino } \\
\text { atraviese dos poblados } \\
\text { con nivel de pobreza } \\
\text { municipal media o } \\
\text { menor. }\end{array}$ & $\begin{array}{l}\text { El } 57 \% \text { de carreteras se } \\
\text { emplazan sobre niveles de } \\
\text { pobreza media-menor } \\
65 \% \text { de los poblados se } \\
\text { concentran en las } \\
\text { carreteras colectoras y } \\
\text { troncales }\end{array}$ \\
\hline $\begin{array}{l}\text { Que pertenezcan a las } \\
\text { carreteras troncales o } \\
\text { colectoras }\end{array}$ & $\begin{array}{l}\text { El 97\% del derecho de vía } \\
\text { de las carreteras en donde } \\
\text { se puede establecer } \\
\text { vegetación, pertenece a } \\
\text { carreteras troncales y } \\
\text { colectoras }\end{array}$ \\
\hline
\end{tabular}

d) Identificación de tramos que cumplen con las características de selección

Se identificaron como válidos para el análisis los tramos de Las Flores-Diriá y Guayacán-Matagalpa, los cuales son representativos de al menos $30 \%$ del sistema ambiental dominante de las carreteras y caminos que constituyen la red vial nacional.

El primer tramo de carretera inicia en el empalme denominado Las Flores, hacia los pueblos blancos de Masaya en las coordenadas UTM 600619.74N, 1323735.55W, discurre por los municipios de: Masaya, Catarina y San Juan de Oriente, hasta llegar a las coordenadas UTM $602474.22 \mathrm{~N}, 1313956.38 \mathrm{~W}$, en el municipio de Diriá, con una longitud de tramo de 12.16 kilómetros.

El segundo tramo de carretera inicia en el empalme de Guayacán en las coordenadas UTM 607636.81N, $1428570.81 \mathrm{~W}$, discurre por el municipio de Matagalpa, atraviesa las comunidades Yaule Arriba, Waswalí Abajo, Las Tejas, y El Pastal hasta terminar en el sitio 
conocido como empalme La Virgen en las coordenadas $615977.04 \mathrm{~N}, 1428377.53 \mathrm{~W}$, con una longitud de 9.24 kilómetros. El área verde en los dos tramos es de $152,460 \mathrm{~m}^{2}$. En la figura 1 se muestra la ubicación de los dos tramos (Jimenez, 2008)

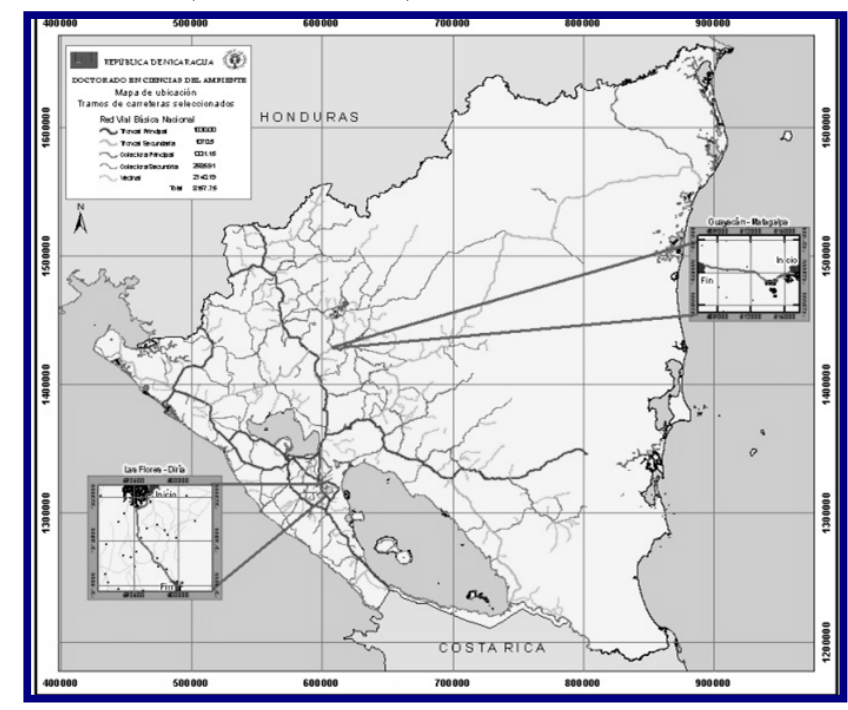

Fig. 1 Mapa de ubicación de los tramos de carreteras seleccionados

\section{Inventario de especies vegetales}

Se ajustaron formatos de campo para el levantamiento de inventarios de árboles y arbustos en el área verde del derecho de vía de los tramos seleccionados (Jiménez, 2008).

Entre los datos principales colectados en el campo, se encuentran: ubicación en coordenadas UTM, cantidad de individuos de una misma especie, el nombre común de cada individuo, diámetro a la altura del pecho (DAP a $1.3 \mathrm{~m}$ ), altura (m), cobertura del dosel, y datos sobre las características del crecimiento de la especie (vitalidad). La recopilación de información se realizó en los meses de septiembre y octubre de 2007.

\section{Análisis de la información colectada}

a) Abundancia y densidad: La abundancia se refiere a una estimación del número de individuos de cada especie presentes, expresadas en términos relativos. La densidad da una idea de la distancia media entre individuos de la especie y para hallar su valor es necesario contar el número de individuos existentes por unidad de superficie (Aguilo et. al, 2004).

La abundancia de las especies fue analizada en las escalas propuestas por: Tansley y Chipp (1926), Braun-
Blanquet, Socher (1933), Hanson (1934) y su correspondencia con valores de densidad de Shimwell (1971). La densidad se determinó dividiendo la cantidad total de individuos por tramo entre $152,460 \mathrm{~m}^{2}$. Para este efecto sólo se contabilizaron las especies que obtuvieran DAP mayores a $10 \mathrm{~cm}$.

b) Diversidad de especies: Es el número de especies dentro de un área determinada.

c) Grado de cubierta: Es el porcentaje de superficie de la unidad de trabajo, cubierto por la proyección horizontal de la vegetación. Se empleó la escala de cinco grados de Margalef, (1974).

d) Dominancia: Indica el espacio de terreno ocupado actualmente por una especie. El área basal es el área de la sección del tronco a la altura de $1.30 \mathrm{~m}$ sobre el nivel del suelo.

e) Composición florística: Se refiere a la lista de especies presente en la comunidad vegetal. Identificando el nombre científico y familia de cada especie vegetal.

e) Vitalidad: Esta característica se refiere al crecimiento de la especie. Se analizaron según la escala de BraunBlanquet (1932).

\section{RESULTADOS Y DISCUSIÓN}

El resultado de la abundancia estudiada desde cuatro escalas diferentes, se resume en la tabla 4. En ella, se muestra la escasa abundancia que tienen los árboles y arbustos que se ubican en los derechos de vía de las carreteras analizadas.

Tabla 4 Abundancia y densidad de árboles y arbustos por tramos de carreteras

\begin{tabular}{|c|c|c|}
\hline Escala & Las Flores-Diría & $\begin{array}{c}\text { Guayacán- } \\
\text { Matagalpa }\end{array}$ \\
\hline $\begin{array}{c}\text { Tansley y Chipp } \\
(1926)\end{array}$ & Rara, $\mathrm{r}$ & Rara, $\mathrm{r}$ \\
\hline $\begin{array}{c}\text { Braun-Blanquet } \\
(1922)\end{array}$ & Muy Escasa & Escasa \\
\hline Socher (1933) & Rara & Poco común \\
\hline Hanson (1934) & Escasa & Escasa \\
\hline Densidad & 0.0046767 & 0.0181556 \\
arboles $/ \mathrm{m}^{2}$
\end{tabular}


Los resultados mostrados en la tabla 4, se encuentran por debajo de los valores de densidad mínimos propuestos para la vegetación por Shimwell, (1971). Esto revela que en el área verde de las carreteras de Nicaragua es mínima la densidad de este estrato vegetal, siendo más frecuente encontrar las especies herbáceas.

A pesar de que la abundancia es escasa, se concentran las especies por tramo de la siguiente manera:

En el tramo Las Flores-Diriá, se agrupan en un $70 \%$ en las especies de: Tabebuia chysantha, Azadirachta indica, Guazuma ulmifolia, Cordia alliodora, Senna siamea, Enterolobium cyclocarpum, Tabebuia rosea, Gliricidia sepium y Delonix regia.

En el tramo de la carretera Guayacán-Matagalpa se determinó que el $75 \%$ de ellas se encuentran concentradas en las siguientes especies: Tecoma stans, Cordia truncalifolia, Gliricidia sepium, Guazuma ulmifolia, Azadirachta indica, y Tabebuia rosea.

Estas especies vegetales tienen cualidades de avanzadas que invaden campos abandonados, toleran sequías, vientos y fuego, pueden crecer en condiciones de suelo extremas y en suelos de poca fertilidad (Barrance et al, 2003)

En la figura 2 se observa que la Tabebuia rosea presiona la estructura de la cuneta revestida deteriorándola; y a su vez, el sistema radicular de esta planta se ve sometida a deformaciones o alteraciones para aprovechar los nutrientes del suelo.

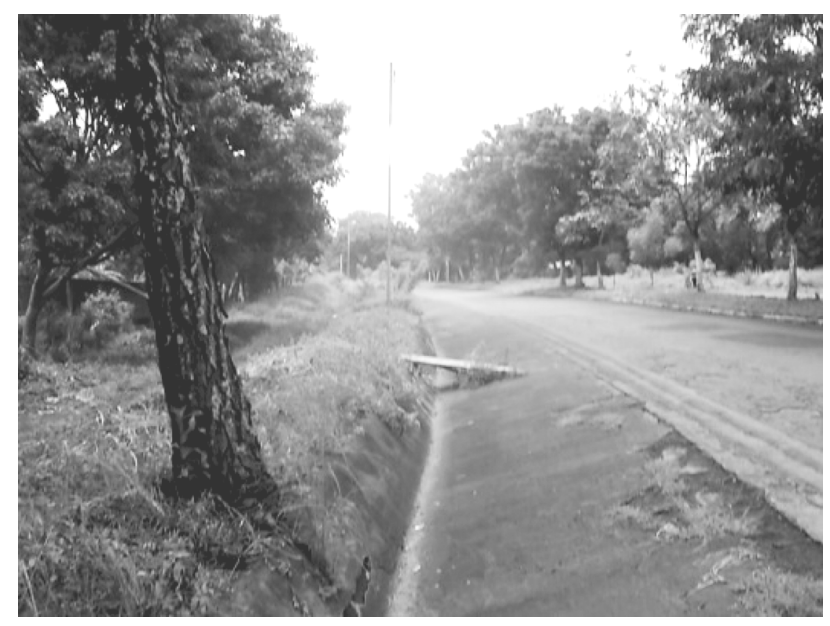

Fig. 2 Adaptación de Tabebuia rosea en estructuras de drenajes en la carretera Las Flores-Diría, 2007. (foto: J.Jiménez)
La riqueza de árboles y arbustos está conformada por 68 especies en total en las dos carreteras, diseminadas en 29 familias.

En la figura 3 se muestran los resultados de la riqueza de las especies presentes por tramos y se observa que es mayor la diversidad de especies y familias en el tramo Guayacán-Matagalpa.

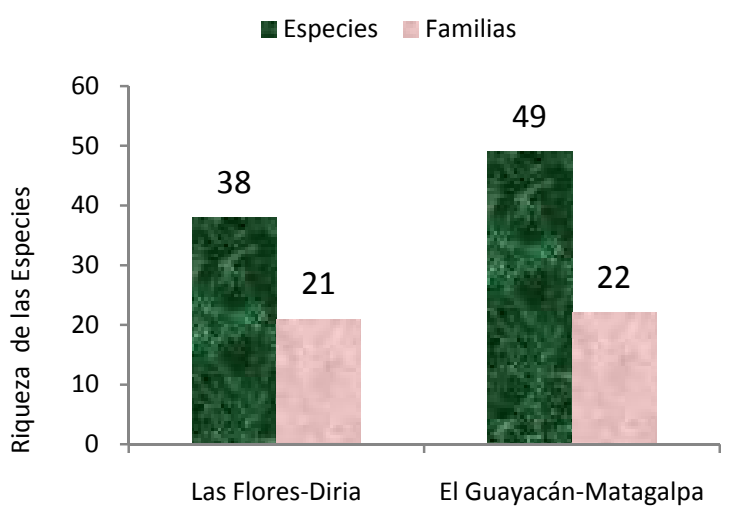

Fig.3 Riqueza de las especies de árboles y arbustos por tramo

Diecinueve especies arbóreas que se distribuyen en catorce familias habitan en los dos tramos estudiados. Cuatro familias tienen más de una especie siendo ellas: Bignoniaceae, Mimosaceae, Fabaceae, y Boraginaceae.

Es notoria la frecuencia de la familia Mimosaceae con diez especies, lo que indica una alta incidencia de plantaciones de árboles en el área.

El grado de cubierta de las especies de árboles y arbustos que pueblan el tramo Guayacán-Matagalpa es del $5 \%$ y el $2 \%$ para la carretera Las Flores-Diriá. Ubicándose en el grado uno (1), rango de $0-5 \%$, en la escala de Margalef, (1974). Lo que indica que este estrato no es el que realiza la función protectora del suelo en esta superficie.

Se identificaron especies dominantes tales como: Delonix regia y Enterolobium cyclocarpum con gran corpulencia, alcanzando más de $100 \mathrm{~cm}$ DAP.

El grosor del tronco de Enterolobium cyclocarpum (DAP), indica la antigüedad de esta planta en el derecho de vía de los tramos analizados, si consideramos un crecimiento anual de $10 \mathrm{~cm}$ a la altura de $1.3 \mathrm{~m}$ en condiciones optimas. 
Coincidiendo con lo aseverado por las investigaciones del Centro Agronómico Tropical de Investigación y Enseñanza (CATIE), el Enterolobium cyclocarpum se encuentra en sitios impactados por el hombre y son remanentes del bosque natural, ya que las plantaciones de esta especie no son muy comunes (Barance et. al, 2003).

Es notorio observar que estas especies (Delonix regia y Enterolobium cyclocarpum) con menor dominancia, se encuentran severamente deformadas en su tronco; tal como se refleja en la figura 4.

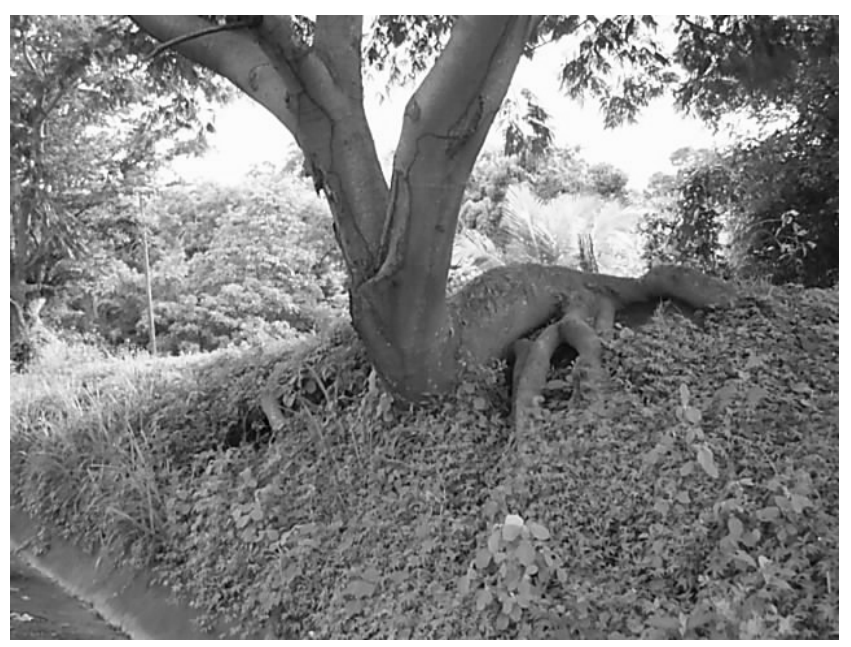

Fig. 4 Tronco y sistemas de raíces deformados del Enterolobium cyclocarpum (foto: J. Jiménez)

El Delonix regia (Caesalpiniaceae) es ornamental en parques y orillas de carreteras, pero no es apreciado por sus raíces superficiales (Ugalde et. al, 1997). Considerando lo anterior, la dominancia de esta especie, las características del sistema radicular y su proximidad con la línea de rodamiento crea un alto riesgo en los derechos de vía de las carreteras estudiadas.

La composición florística de los árboles y arbustos se describen en la tabla 5, indicando con una equis (X) la que corresponde a cada tramo de carretera.

La vitalidad de las especies osciló entre normal y vigoroso en el tramo de Las Flores-Diriá; y de normal a débil en la carretera Guayacán-Matagalpa.
Tabla 5 Composición florística de los árboles y arbustos por tramos

\begin{tabular}{|c|c|c|c|}
\hline & & Tra & \\
\hline Nombre científico & Familia & 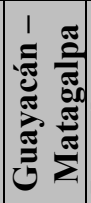 & 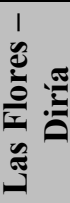 \\
\hline Acacia farnesiana & Mimosaceae & $X$ & \\
\hline Acacia hindis Beth & Mimosaceae & $X$ & \\
\hline $\begin{array}{c}\text { Acacia } \\
\text { pennatula(Schlecht) }\end{array}$ & Mimosaceae & $X$ & \\
\hline $\begin{array}{c}\text { Andira inermes ( } W . \\
\text { Wright) }\end{array}$ & Fabaceae & & $X$ \\
\hline Azadirachta indica & Meliaceae & $X$ & $X$ \\
\hline Bombacopsis quinata & Bombacaceae & $X$ & \\
\hline Bursera simarouba & Bignoniaceae & $X$ & \\
\hline Byrsonima crassifolia & Malpighiaceae & $X$ & $X$ \\
\hline Caesalpinia pulcherrima & Mimosaceae & $X$ & \\
\hline Caesalpinia velutina & Caesalpiniaceae & $X$ & \\
\hline $\begin{array}{c}\text { Calycophyllum } \\
\text { candidisimun (Vahl) Dc }\end{array}$ & Rubiaceae & $X$ & $X$ \\
\hline Cassia fistula & Caesalpiniaceae & $X$ & $X$ \\
\hline Cassia grandis & Caesalpiniaceae & $X$ & \\
\hline Cecropia peltata $L$. & Moraceae & & $X$ \\
\hline Cedrela adorata L. & Meliaceae & & $X$ \\
\hline Ceiba pentandra & Bombacaceae & & $X$ \\
\hline Chrysophyllum Cainito & Sapotaceae & $X$ & \\
\hline Citrus X aurantifolia & Rutaceae & & $X$ \\
\hline Citrus $X$ aurantium & Rutaceae & & $X$ \\
\hline Coccoloba floribunda & Polygonaceae & $X$ & \\
\hline Cocus nucifera $L$. & Arecaceae & & $X$ \\
\hline Cordia alliodora & Boriginaceae & & $X$ \\
\hline Cordia dentada & Boraginaceae & $X$ & $X$ \\
\hline Cordia truncatifolia & Boraginaceae & $X$ & $X$ \\
\hline Crescentia alata & Bignoniaceae & $X$ & $X$ \\
\hline Cupressus benthamii & Cupressaceae & $X$ & \\
\hline Delonix regia & Fabaceae & $X$ & $X$ \\
\hline $\begin{array}{l}\text { Esterolobium } \\
\text { cyclocarpum }\end{array}$ & Mimosaceae & $X$ & $X$ \\
\hline $\begin{array}{c}\text { Eucalyptus } \\
\text { camaldulensis }\end{array}$ & Myrtaceae & $X$ & $X$ \\
\hline Ficus benjamina & Moraceae & $X$ & $X$ \\
\hline Ficus cotinifolia & Moraceae & $X$ & \\
\hline Gliricidia sepium & Fabaceae & $X$ & $X$ \\
\hline Guaizum sanctum & Zygophyllaceae & $X$ & \\
\hline Guazuma ulmifolia & Sterculiaceae & $X$ & $X$ \\
\hline Hamelia patens & Rubiaceae & $X$ & \\
\hline $\begin{array}{l}\text { Inga vera Willd. Ssp. } \\
\text { Spuria (Willd) }\end{array}$ & Mimosaceae & & $X$ \\
\hline
\end{tabular}




\begin{tabular}{|c|c|c|c|}
\hline \multirow[b]{2}{*}{ Nombre científico } & \multirow[b]{2}{*}{ Familia } & \multicolumn{2}{|c|}{ Tramo } \\
\hline & & 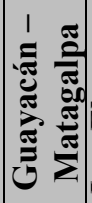 & 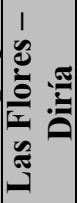 \\
\hline Jacquinia aurantiaca & Theophrastaceae & $X$ & \\
\hline $\begin{array}{c}\text { Leucaena leucocephala } \\
\text { (Lam) de Wit }\end{array}$ & Mimosaceae & $X$ & \\
\hline Leucaena shanoni & Mimosaceae & $X$ & \\
\hline $\begin{array}{c}\text { Lonchocarpus } \\
\text { parviflorus Benth }\end{array}$ & Fabaceae & $X$ & \\
\hline lysiloma aurifum & Mimosaceae & $X$ & \\
\hline Magnifera indica & Anacardiaceae & $X$ & $X$ \\
\hline Melia azedarach & Meliaceae & $X$ & \\
\hline Melicocus bijugafus & Sapindaceae & $X$ & $X$ \\
\hline Myrospermun frutescens & Fabaceae & $X$ & \\
\hline Muntingia Calabura l. & Elaeocarpaceae & & $X$ \\
\hline Persea Americana Mill & Lauraceae & & $X$ \\
\hline Phyllostylon brasiliensis & Ulmaceae & $X$ & \\
\hline Picus insipida Will & Moraceae & & $X$ \\
\hline Pisonia aculeata & Fabaceae & & $X$ \\
\hline Pithecellobium saman & Mimosaceae & $X$ & $X$ \\
\hline Plumeria acutifolia & Apocynaceae & & $X$ \\
\hline Psidium guajava & Myrtaceae & $X$ & \\
\hline Rehdera trinervis & Verbenaceae & $X$ & \\
\hline Sapindus saponaria $L$ & Sapindaceae & & $X$ \\
\hline Senna otomaria $(L)$ & Caesalpiniaceae & $X$ & \\
\hline Senna siamea (Lam.) & Caesalpiniaceae & & $X$ \\
\hline Simarouba glauca & Simaroubaceae & & $X$ \\
\hline $\begin{array}{c}\text { Spathodea campanulata } \\
\text { Beauv }\end{array}$ & Bignoniaceae & & $X$ \\
\hline Spondias porpurea L. & Anacardiaceae & $X$ & \\
\hline Swietenia humilis & Meliaceae & $X$ & \\
\hline Tabebuia Chrisantha & Bignoniaceae & & $X$ \\
\hline Tabebuia rosea & Bignoniaceae & $X$ & $X$ \\
\hline Tecoma stans & Bignoniaceae & $X$ & $X$ \\
\hline Terminalia amazonia & Combretaceae & $X$ & $X$ \\
\hline Terminalia catappa $L$. & Combretaceae & $X$ & \\
\hline $\begin{array}{c}\text { Trema micratha }(L) \\
\text { Blume }\end{array}$ & Ulmaceae & $X$ & \\
\hline $\begin{array}{c}\text { Zyzyphus guatemalensis } \\
\text { Hemsl }\end{array}$ & Rhamnaceae & $X$ & \\
\hline
\end{tabular}

\section{CONCLUSIONES}

Se concluye que las especies de árboles que habitan en las áreas verdes de los derechos de vía de las carreteras analizadas son especies ruderales, o remanentes de bosques riparios, siendo frecuente encontrar: Tabebuia rosea, Gliricidia sepium, Azadirachta indica, Guazuma ulmifolia, Cordia alliodora, Senna siamea, y Enterolobium cyclocarpum.

Sin embargo, estos árboles se localizan con abundancia escasa, mínima densidad, bajo porcentaje de cobertura vegetal, y razonable diversidad de especies que se deben de proteger.

Además, se sugiere el estudio de las especies herbáceas que tienen mayor cobertura en el área verde de las carreteras.

\section{REFERENCIAS}

Aguilo, M., Aramburu, M., Blanco, A., Calatayud, T., Carrasco, R., Castilla, G., Castillo, V., Ceñal, M., Cifuentes, P., Diaz, A., Escribano, R., Escribano, M., Frutos, M., Galiana, F., Garcia, A., Glaria, G., Gonzalez, S., Gonzalez, C., \& Iglesias, E. (2004) Guía para la elaboración de estudios del medio fisico. Contenido y Metodologías. pp. 383-430. Ministerio de Medio Ambiente. España.

Barrance, A., Beer, J., Boshier, D., Chamberlain, J., Corder, J., Detlefsen, G., Finegan, B., Galloway, G., Gómez, M., Gordon, J., Hands, M., Hellin, J., Hughes, C., Ibrahim, M., Kass, D., Leaker, R., Mesén, F., Montero, M., Rivas, C., Somarriba, E., Stewart, J., \& Pennington, T. (2003). Árboles de Centroamérica. pp 1077. Centro Agronómico Tropical de Investigación y Enseñanza CATIE, Costa Rica.

EDICO, Ingenieros Consultores (1998), Estudios y Diseños para la Rehabilitación de la carretera Las Flores-Nandaime. I-VIII volúmenes Ministerio de Transporte e Infraestructura. Managua, Nicaragua.

INOCSA, Ingenieros Consultores (2003), Estudios y Diseños para la rehabilitación de la carretera SébacoMatagalpa. I-X volúmenes Ministerio de Transporte E Infraestructura. Managua, Nicaragua.

Jiménez, J. (2008), Instrumental Metodológico para el Manejo Ambiental de la Vegetación en Proyectos de Carreteras Troncales de Nicaragua Tesis para optar al grado de Doctor en Ciencias del Ambiente. Universidad Nacional de Ingeniería, UNI. Managua, Nicaragua.

Ministerio del Ambiente y Recursos Naturales, Servicio Forestal Nacional, MARENA SFN (1995) Especies 
para Reforestación en Nicaragua. pp.109. Managua, Nicaragua.

Ministerio del Ambiente y Recursos Naturales, MARENA- INAFOR (2002) Guia de Especies Forestales de Nicaragua. pp. 304. Managua, Nicaragua.

Ministerio de Obras Públicas y Transporte, MOPT, (1991) Guía Metodológica para la elaboración de estudios de impacto ambiental: 1. Carreteras $y$ Ferrocarriles. pp. 1-161. España.

Milán, J. (2004) Manual de Estudios Ambientales para la Planificación y los proyectos de Desarrollo. pp. 500 Universidad Nacional de Ingeniería UNI-PEAUT. Managua.

Ministerio de Transporte e Infraestructura, MTI (2005) Red Vial de Nicaragua 2004. Pp. 25. Dirección General de Planificación - MTI. Managua, Nicaragua.

Ministerio de Transporte e Infraestructura, MTI (2000). Plan Nacional de Transporte 2000-2020. CORASCO Y CISCONCO. VI Volúmenes. Dirección General de Planificación-MTI. Managua, Nicaragua.

Pérez, M., Sotelo, M., Ramírez, F., Ramírez, I., López, A., \& Siria, I. (2005), Conservación de la biodiversidad en Sistemas silvopastoriles de Matiguas y Rio Blanco (Matagalpa, Nicaragua). AEET, Ecosistemas 15 (3) (en: http://www.revistaecosistemas.net)

Salas, J. (2002) Biogeografia de Nicaragua, pp. 547. Instituto Nacional Forestal (INAFOR). Managua.
Salas, J. (1993) Árboles de Nicaragua, pp. 338. Instituto Nicaragüense de Recursos Naturales y del Ambiente (IRENA). Managua, Nicaragua.

Servicio Forestal Nacional (1992) Árboles Forestales Útiles para su Propagación. pp. 238 Instituto Nicaragüense de Recursos Naturales y del Ambiente (IRENA), Managua Nicaragua.

Ugalde, L., \& Otárola, A., (1997). Resultado de 10 años de Investigación Silvicultural del Proyecto MADELEÑA de Nicaragua, pp. 174 Centro Agronómico Tropical de Investigación y Enseñanza CATIE.

Zietlow, J. (2002) Sinopsis de Manuales de Construcción y Mantenimiento Vial en América Latina y el Caribe, versión 1. pp.102. Washington DC.

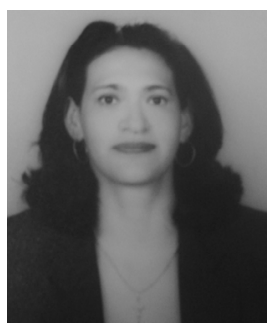

Juliana Jiménez se graduó de Licenciada en Ecología y Recursos Naturales en la Universidad Centroamericana (UCA) en 1990. En la Universidad Nacional de Ingeniería- Programa de Estudios Ambientales Urbanos Territoriales (UNI-PEAUT), obtuvo su título de Especialista en Gestión y Evaluación de Impacto Ambiental en 2002, continuó sus estudios de Maestría en Planificación y Administración Ambiental de Proyectos, 2005 y realizó estudios de Doctor en Ciencias del Ambiente, 2008. 